\title{
A new algorithm for the detection of seismic quiescence: introduction of the RTM algorithm, a modified RTL algorithm
}

\author{
Toshiyasu Nagao ${ }^{1}$, Akihiro Takeuchi ${ }^{1}$, and Kenji Nakamura ${ }^{2}$ \\ ${ }^{1}$ Earthquake Prediction Research Center, Institute of Oceanic Research and Development, Tokai University, Shizuoka, Japan \\ ${ }^{2}$ Graduate School of Marine Science and Technology, Tokai University, Shizuoka, Japan
}

(Received July 8, 2010; Revised December 16, 2010; Accepted December 19, 2010; Online published March 4, 2011)

\begin{abstract}
There are a number of reports on seismic quiescence phenomena before large earthquakes. The RTL algorithm is a weighted coefficient statistical method that takes into account the magnitude, occurrence time, and place of earthquake when seismicity pattern changes before large earthquakes are being investigated. However, we consider the original RTL algorithm to be overweighted on distance. In this paper, we introduce a modified RTL algorithm, called the RTM algorithm, and apply it to three large earthquakes in Japan, namely, the Hyogoken Nanbu earthquake in 1995 ( $\left.M_{\mathrm{JMA}} 7.3\right)$, the Noto Hanto earthquake in $2007\left(M_{\mathrm{JMA}} 6.9\right)$, and the IwateMiyagi Nairiku earthquake in 2008 ( $\left.M_{\mathrm{JMA}} 7.2\right)$, as test cases. Because this algorithm uses several parameters to characterize the weighted coefficients, multiparameter sets have to be prepared for the tests. The results show that the RTM algorithm is more sensitive than the RTL algorithm to seismic quiescence phenomena. This paper represents the first step in a series of future analyses of seismic quiescence phenomena using the RTM algorithm. At this moment, whole surveyed parameters are empirically selected for use in the method. We have to consider the physical meaning of the "best fit" parameter, such as the relation of $\Delta C F S$, among others, in future analyses.

Key words: Seismicity, seismic quiescence, RTM, RTL, precursor.
\end{abstract}

\section{Introduction}

Several methods are currently available to diagnose future seismic activity, including the $Z$-value (Wyss and Habermann, 1988) and ETAS (e.g., Ogata, 2006), or predict major earthquakes, such as M8 (Keilis-Borok and Kossobokov, 1990), RTP (Shebalin et al., 2004), and Hotspot (Rundle et al., 2002). In addition, there are many reports of seismic activity decreasing prior to the occurrence of major earthquakes in or around the focal region (e.g., Mogi, 1979; Wyss et al., 1981; Kisslinger, 1988; Wyss and Habermann, 1988; Wiemer and Wyss, 1994). This latter phenomenon, called seismic quiescence, is expected to provide useful information for earthquake prediction. In many of the above reports the authors frequently claim that the seismic quiescence and activation occurred simultaneously. Matsumura (2005) has also modeled these phenomena as occurring simultaneously under redistribution of the tectonic stress, resulting in a net quiescence.

To detect the occurrence of quiescence, Sobolev and Tyupkin (1997, 1999) proposed a weighted coefficient method called the RTL algorithm. The basic assumption of this method is that each prior event has some influence on the main event under investigation and that this influence weight varies as described by the formulae below. The value of $R T L$, which is supposed to represent the state of seismicity at the position $(x, y, z)$ at time $t$, is the product

Copyright (C) The Society of Geomagnetism and Earth, Planetary and Space Sciences (SGEPSS); The Seismological Society of Japan; The Volcanological Society of Japan; The Geodetic Society of Japan; The Japanese Society for Planetary Sciences; TERRAPUB.

doi:10.5047/eps.2010.12.007 of three dimensionless factors: $R(x, y, z, t), T(x, y, z, t)$, and $L(x, y, z, t)$. The factors are defined as:

$$
\begin{aligned}
& R(x, y, z, t)=\left[\sum_{i=1}^{n} \exp \left(-\frac{r_{i}}{r_{0}}\right)\right]-R_{\mathrm{tr}}(x, y, z) \\
& T(x, y, z, t)=\left[\sum_{i=1}^{n} \exp \left(-\frac{t-t_{i}}{t_{0}}\right)\right]-T_{\mathrm{tr}}(x, y, z), \\
& L(x, y, z, t)=\left[\sum_{i=1}^{n}\left(\frac{l_{i}}{r_{i}}\right)\right]-L_{\mathrm{tr}}(x, y, z)
\end{aligned}
$$

where $r_{i}$ is the distance between the position and the $i$ th earthquake's hypocenter, $r_{0}$ is the characteristic distance, $R_{\mathrm{tr}}(x, y, z)$ is the trend of $R(x, y, z)$ in the calculation period, $t_{i}$ is the occurrence time of the $i$ th earthquake, $t_{0}$ is the characteristic time-span, $T_{\mathrm{tr}}(x, y, z)$ is the trend of $T(x, y, z)$ in the calculation period, $l_{i}$ is the $i$ th earthquake's rupture dimension (= fault length) in kilometers obtained from the relation with the $i$ th earthquake's magnitude $M_{i}: \log l_{i}=0.5 M_{i}-1.8$ (Kasahara, 1981), and $L_{\mathrm{tr}}(x, y, z)$ is the trend of $L(x, y, z)$ in the calculation period. The integer $n$ is the number of earthquake events that satisfy the following criteria:

$$
\begin{aligned}
& M_{i} \geq M_{\min }, \\
& r_{i} \leq R_{\max }=k_{\mathrm{r}} r_{0}, \\
& t-t_{i} \leq T_{\max }=k_{\mathrm{t}} t_{0},
\end{aligned}
$$

where $M_{\min }$ is the cut-off magnitude ensuring the completeness of the earthquake catalog after declustering, and $R_{\max }$ and $T_{\max }$ are the cut-off distance and time interval, respectively. In the past, almost all studies adopted 2 for $k_{\mathrm{r}}$ and 


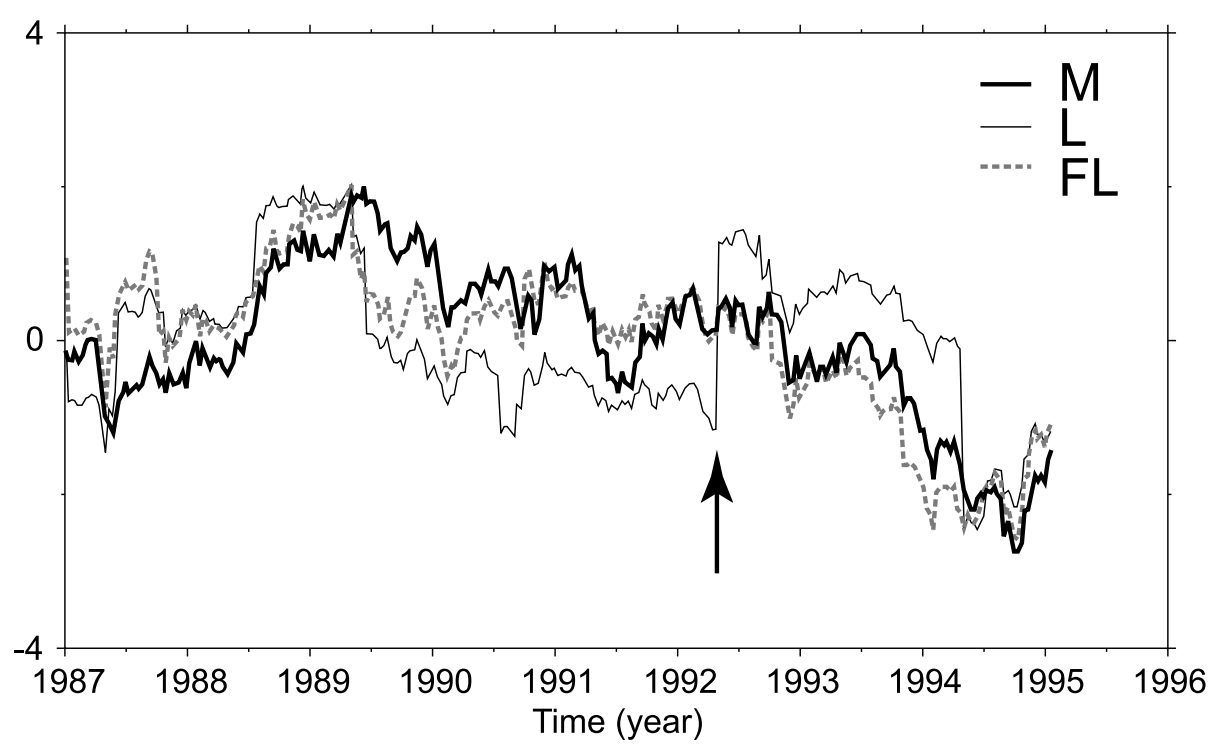

Fig. 1. Time variation of factors $L, F L$, and $M$ at the epicenter $\left(135.04^{\circ} \mathrm{E}, 34.59^{\circ} \mathrm{N}\right)$ of the Hyogo-ken Nanbu Earthquake in $1995(M \mathrm{JMA} 7.3)$ in southwest Japan. The arrow showed an abrupt shift of the factor $L$ in April 1992. Thick, thin and dotted lines show $M, L$ and $F L$, respectively.

$k_{\mathrm{t}}$. The three factors $(R, T$, and $L)$ actually used are, after normalization by their standard deviations, $\sigma_{R}, \sigma_{T}$, and $\sigma_{L}$, respectively. Therefore, the calculated $R T L$ value is in the unit of the standard deviation $\left(\sigma=\sigma_{R} \sigma_{T} \sigma_{L}\right)$. Detailed mathematical and statistical descriptions of the RTL method are well documented in Huang (2006).

Positive values of $R T L$ indicate seismic activation, while negative values indicate quiescence. Huang et al. (2001) showed that the $R T L$ value was approximately -7 to -10 at the epicenter of the Hyogo-ken Nanbu earthquake in $1995\left(M_{\mathrm{JMA}} 7.3\right.$, the so-called 1995 Kobe earthquake about 6 months before the earthquake, where $M_{\mathrm{JMA}}$ is the earthquake magnitude defined by the Japan Meteorological Agency (JMA)). Huang and Nagao (2002) showed a clear spatial and temporal quiescence pattern at around the epicenter of the 2000 Tottori earthquake ( $M_{\mathrm{JMA}} 7.3$, Japan). Huang (2008) recently applied the RTL algorithm to the 2008 Wenchuan earthquake $\left(M_{\mathrm{S}} 8.0\right.$, China). Wyss et al. (2004) applied both the $Z$ value and the RTL methods to two major earthquakes in Sakhalin and concluded that the two methods yield almost identical results, strongly suggesting that the observed precursory quiescence anomalies are robust and real.

As shown in Eqs. (1) and (3), $r_{i}$ is used to calculate both $R$ and $L$. This dual appearance of $r_{i}$ seems to be in contradiction to the original concept of the RTL algorithm, i.e., the equi-importance of space and time. To remedy this situation, we have modified the RTL algorithm and apply this new algorithm (the RTM algorithm) to three Japanese earthquakes in Section 4.

Both the RTL and RTM algorithms contain a number of adjustable parameters, namely, $T_{0}, T_{\max }, R_{0}, R_{\max }$, and others. In the past, the most suitable set of parameters was retrospectively chosen by a trial and error method. Seismic quiescence phenomena do not always result in a large earthquake. Sometimes nothing happens. Therefore, it is essential that seismologists screen out, as much as possible, false (artificial) quiescence. To this end, we perform a mul- tiparameter survey test (more than ten parameter sets) with the aim of obtaining a good understanding of the nature of seismic quiescence phenomena.

\section{RTM Algorithm}

The factor $L$ of the RTL algorithm includes $r_{i}$ in its definition (Eq. (3)). In this section, we try to explain the RTL algorithm and the advantages of the RTM algorithm using the case of the Hyogo-ken Nanbu earthquake in 1995 in Japan ( $\left.M_{\mathrm{JMA}} 7.3\right)$. Figure 1 shows the time variation of $L$ at the epicenter $\left(135.04^{\circ} \mathrm{E}, 34.59^{\circ} \mathrm{N}\right)$ of the main shock. Here, $L$ is normalized by its standard deviation $\sigma_{L}$. The JMA earthquake catalog is used. The original data set $\left(M_{\mathrm{JMA}} \geq\right.$ $1.5)$ is declustered using the JMA's standard program $(\mathrm{H}$. Takayama, personal communication). This decluster program is classified as a link algorithm (e.g., Frohlich and Davis, 1990). Earthquakes that occurred within a certain epicentral distance within a certain day from a prior earthquake are judged to be aftershocks of this earthquake. The JMA has selected $3 \mathrm{~km}$ and 7 days in this algorithm (A. Yoshida, personal communication), and we adopt these values in this study. We call this the " $3 \mathrm{~km} / 7$ days" routine hereafter.

There is a strange shift of $L$ in April 1992, as shown by an arrow in Fig. 1. A careful survey of the used data reveal that an earthquake occurred very close to the epicenter of the main shock at that time. The very small $r_{i}$ in Eq. (3) quite naturally makes $l_{i} / r_{i}$ and $L$ very large. Here, note that the completeness of the earthquake catalog is not considered in Fig. 1 because the aim of this figure is to show the effects on the factor $L$.

To avoid this effect, which is due to the dual appearance of $r_{i}$, we introduce new factors $F L$ (fault length) and $M$ (magnitude) as defined by:

$$
F L(x, y, z, t)=\left[\sum_{i=1}^{n} l_{i}\right]-F L_{\mathrm{tr}}(x, y, z),
$$




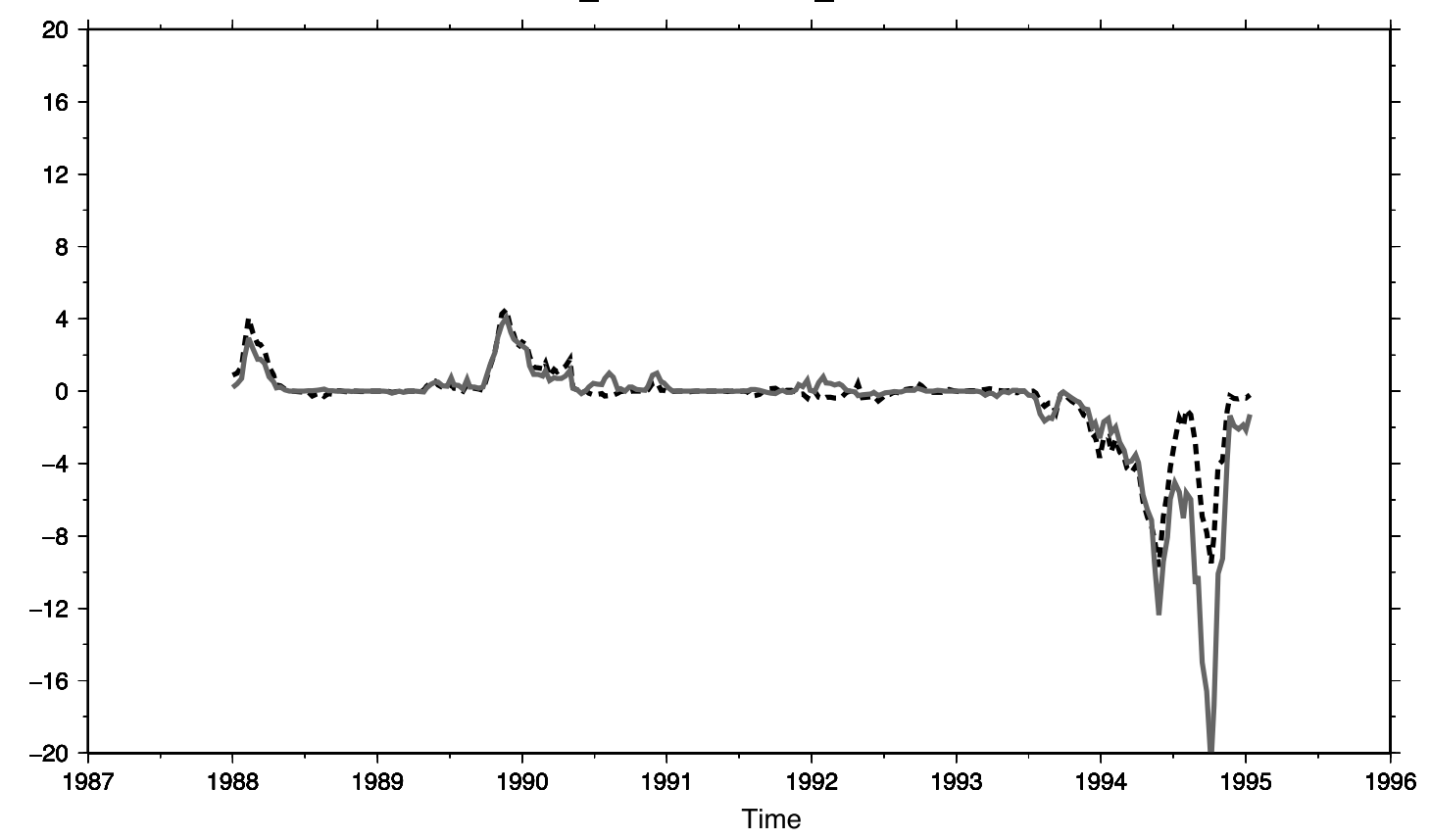

Fig. 2. Time variations of $R T L$ (dotted line) and $R T M$ (solid line) under the parameter set $\# 15$ at the epicenter $\left(135.04^{\circ} \mathrm{E}, 34.59^{\circ} \mathrm{N}\right)$ of the $1995 \mathrm{Kobe}$ Earthquake $\left(M_{\mathrm{JMA}} 7.3\right)$ in southwest Japan.

$$
M(x, y, z, t)=\left[\sum_{i=1}^{n} M_{i}\right]-M_{\mathrm{tr}}(x, y, z),
$$

where $F L_{\mathrm{tr}}(x, y, z)$ is the trend of $F L(x, y, z)$ in the calculation period, $M_{i}$ is the $i$ th earthquake's magnitude, and $M_{\mathrm{tr}}(x, y, z)$ is the trend of $M(x, y, z)$ in the calculation period. These factors do not include $r_{i}$. Figure 1 also shows the time variations of $F L$ and $M$. Here, $F L$ and $M$ are normalized by their standard deviation $\left(\sigma_{F L}\right.$ and $\left.\sigma_{M}\right)$. They do not show any abrupt shift in their values. Since the degree of accuracy of $M$ and $F L$ are basically the same, in this paper, we adopt the dimensionless $M$ as a new factor.

The new indicator $R T M$ is defined as the product of three dimensionless factors, namely, $R, T$, and $M$. We recognize seismic quiescence when the RTM (and $R T L$ ) values are $\leq-8$ (darker shading in all Tables). This number $(-8)$ comes from the product of -2 sigma anomalies in the three factors $(R, T$, and $M)$. We recognize seismic quasi-quiescence when the $R T M$ (and $R T L$ ) values are $\leq-6$ (lighter shading in Tables 2 and 3).

\section{Completeness Check of the Seismic Catalog}

It is important to check the completeness of earthquake catalogs before evaluating the seismic activity. Hi-net, which is the new dense seismic station network in Japan (e.g. Obara 2003) established after the 1995 Kobe Earthquake, has made the detectability of inland earthquakes in Japan very high. The way of confirmation of the completeness itself has been an interesting study, and various ways have been proposed. One of the most sophisticated methods is a probability-based magnitude of completeness (PMC) method (e.g., Nanjo et al., 2010a). However, compared with Gutenberg-Richter (G-R) based methods (e.g., Woessner and Wiemer, 2005), applying the PMC method is more difficult because it requires detailed knowledge about the network and its setup etc.

In this study, we evaluate $M_{\mathrm{c}}$, the lower limit of magnitude for the catalog completeness, from the GutenbergRichter plots of the initial 1-year data in the calculation period. As a result, we found $M_{\mathrm{c}}=2.5$ for the 1995 Kobe Earthquake when we used the catalog after 1987 (see figure 2 of Huang et al., 2001). In contrast, Huang et al. (2001) determined $M_{\mathrm{c}}=3.0$ because they started the calculation in 1978. For other two earthquakes (the Noto Hanto Earthquake in 2007 ( $\left.M_{\mathrm{JMA}} 6.9\right)$ and the IwateMiyagi Nairiku Earthquake in 2008 ( $\left.M_{\text {JMA }} 7.2\right)$ ), we found $M_{\mathrm{c}}=1.5$.

\section{Parameter Survey}

The RTL and RTM algorithms have a number of adjustable parameters. The most suitable set of parameters is chosen retrospectively through an extensive parameter survey. In this section, we show how the RTL and RTM algorithms work by using the examples of their application to three major earthquakes in Japan.

The devastating 1995 Kobe earthquake $\left(M_{\mathrm{JMA}} 7.3\right)$ occurred in southwest Japan on January 17, 1995. As already mentioned, Huang et al. (2001) showed the existence of seismic quiescence before this earthquake by the original RTL algorithm. To verify the effectiveness of the new RTM algorithm, we applied it to this earthquake as an example.

Earthquake data from JMA are declustered with the " $3 \mathrm{~km} / 7$ days" routine. Figure 2 shows the time variations of $R T L$ and $R T M$ in parameter set \#15 of Table 1 at the epicenter $\left(135.04^{\circ} \mathrm{E}, 34.59^{\circ} \mathrm{N}\right)$. The calculation was made during the period from January 1, 1988 to 1 day before the occurrence of the earthquake. Both RTL and RTM indicate abnormal decreases before the earthquake, which acquire 
Table 1. Results of parameter survey in RTL and RTM algorithms at the epicenter of the 1995 Kobe Earthquake (MJMA 7.3).

\begin{tabular}{|c|c|c|c|c|c|c|c|c|c|c|c|}
\hline \multirow{2}{*}{ No. } & \multicolumn{5}{|c|}{ Parameters used for calculation } & \multicolumn{6}{|c|}{$\begin{array}{l}\text { Values at the minimum } R T L \text { or } R T M \\
\text { within } T_{\max } \text { from the occurrence time }\end{array}$} \\
\hline & $\begin{array}{l}R_{0} \\
(\mathrm{~km})\end{array}$ & $\begin{array}{l}R_{\max } \\
(\mathrm{km})\end{array}$ & $\begin{array}{l}T_{0} \\
\text { (year) }\end{array}$ & $\begin{array}{l}T_{\max } \\
\text { (year) }\end{array}$ & $M_{\min }$ & $R$ & $T$ & $L$ & $M$ & $R T L$ & $R T M$ \\
\hline 1 & 25 & 50 & 0.5 & 1 & 1.5 & 1.51 & 1.94 & -0.92 & 0.94 & -2.69 & 1.34 \\
\hline 2 & 25 & 75 & 0.5 & 1 & 1.5 & 1.75 & 1.41 & -0.78 & 1.26 & -1.93 & 2.23 \\
\hline 3 & 25 & 100 & 0.5 & 1.5 & 1.5 & 1.92 & 1.80 & -0.82 & 0.17 & -2.83 & 0.02 \\
\hline 4 & 25 & 100 & 0.5 & 2 & 1.5 & 1.50 & 1.79 & -1.06 & -0.88 & -2.83 & -0.67 \\
\hline 5 & 25 & 100 & 0.5 & 2 & 2 & 2.08 & 3.45 & -0.61 & -1.12 & -4.36 & -0.46 \\
\hline 6 & 25 & 100 & 0.5 & 2 & 2.5 & -1.60 & -2.73 & -2.45 & -2.05 & -10.70 & -8.96 \\
\hline 7 & 50 & 100 & 1 & 2 & 1.5 & 1.79 & 1.86 & -1.06 & -0.88 & -3.53 & -0.63 \\
\hline 8 & 50 & 100 & 1 & 2 & 2 & 2.53 & 2.80 & -0.61 & -1.21 & -4.30 & -0.80 \\
\hline 9 & 50 & 100 & 1 & 2 & 2.5 & -2.37 & -2.61 & -2.17 & -2.74 & -13.39 & -16.95 \\
\hline 10 & 50 & 150 & 0.5 & 2 & 1.5 & 1.56 & 1.40 & -1.46 & -0.55 & -3.20 & -0.30 \\
\hline 11 & 50 & 150 & 1 & 3 & 1.5 & -0.89 & -0.59 & -1.95 & -0.52 & -1.03 & -0.33 \\
\hline 12 & 50 & 200 & 1 & 4 & 1.5 & 1.26 & 1.32 & -0.91 & -0.23 & -1.52 & -0.07 \\
\hline 13 & 75 & 150 & 1 & 2 & 2 & 2.30 & 2.97 & -0.58 & -1.05 & -3.98 & -0.24 \\
\hline 14 & 75 & 225 & 1 & 3 & 2 & 1.53 & 1.15 & -0.53 & 0.38 & -0.94 & -0.03 \\
\hline 15 & 75 & 225 & 1 & 3 & 2.5 & -2.32 & -2.54 & -1.63 & -2.97 & -9.71 & -20.48 \\
\hline 16 & 75 & 300 & 1 & 4 & 3 & -2.58 & -2.67 & -2.11 & -2.56 & -14.60 & -17.68 \\
\hline
\end{tabular}

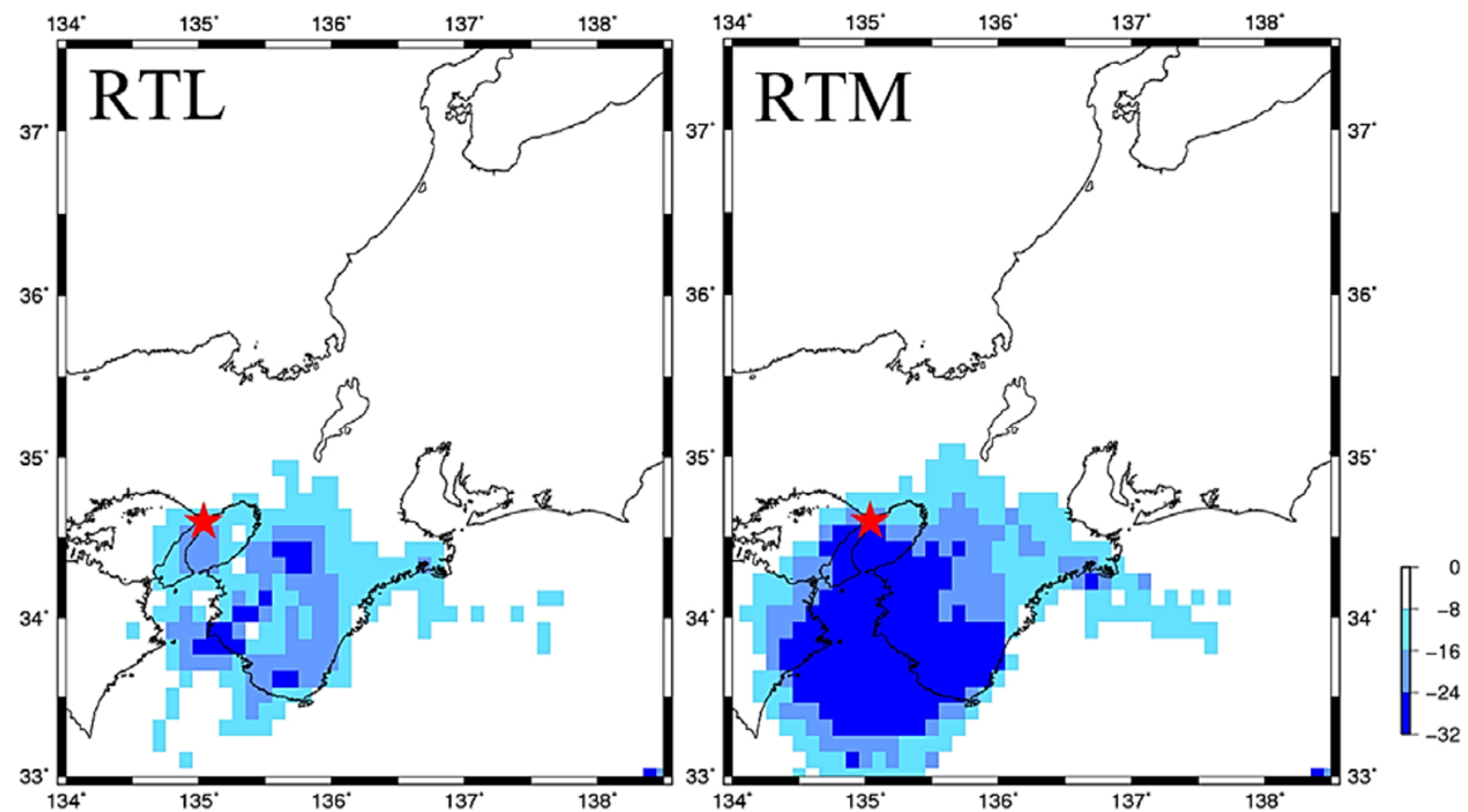

Fig. 3. Spatial distributions of the seismic quiescence in the RTL and RTM algorisms before the 1995 Kobe Earthquake. Figures represent the most quiescent period at the end of September, 1994 (about 3.5 months before the main shock). Stars show the epicenter.

a minimum within $T_{\max }$ (see Eq. (6)) from the occurrence time. The variations are similar for $R T L$ and $R T M$, but the abnormal decreases are more conspicuous for RTM than for $R T L$. Table 1 shows the minimum RTL and RTM values for various parameter sets. Based on these data, clear quiescence (4 cases; shaded column) appears only for large $M_{\min }$ alluding to the incompleteness of the seismic catalog. At the time of the Kobe earthquake, the Hi-net system was not 
Table 2. Results of parameter survey in RTL and RTM algorithms at the epicenter of the Noto Hanto Earthquake in 2007 ( $\left.M_{\mathrm{JMA}} 6.9\right)$.

\begin{tabular}{|c|c|c|c|c|c|c|c|c|c|c|c|}
\hline \multirow{2}{*}{ No. } & \multicolumn{5}{|c|}{ Parameters used for calculation } & \multicolumn{6}{|c|}{$\begin{array}{l}\text { Values at the minimum } R T L \text { or } R T M \\
\text { within } T_{\max } \text { from the occurrence time }\end{array}$} \\
\hline & $\begin{array}{l}R_{0} \\
(\mathrm{~km})\end{array}$ & $\begin{array}{l}R_{\max } \\
(\mathrm{km})\end{array}$ & $\begin{array}{l}T_{0} \\
\text { (year) }\end{array}$ & $\begin{array}{l}T_{\max } \\
\text { (year) }\end{array}$ & $M_{\min }$ & $R$ & $T$ & $L$ & $M$ & $R T L$ & $R T M$ \\
\hline 1 & 25 & 50 & 0.5 & 1 & 1.5 & 0.56 & -0.46 & 0.68 & 0.80 & -0.18 & -0.21 \\
\hline 2 & 25 & 75 & 0.5 & 1 & 1.5 & 1.03 & -0.90 & 0.89 & -1.75 & -0.83 & -1.84 \\
\hline 3 & 25 & 100 & 0.5 & 1.5 & 1.5 & -1.04 & -2.73 & -0.79 & -2.74 & -2.25 & -7.75 \\
\hline 4 & 25 & 100 & 0.5 & 2 & 1.5 & 1.39 & -1.67 & 1.61 & -2.02 & -3.72 & -7.11 \\
\hline 5 & 25 & 100 & 0.5 & 2 & 2 & 1.63 & -1.60 & 1.44 & -1.73 & -3.76 & -3.90 \\
\hline 6 & 25 & 100 & 0.5 & 2 & 2.5 & 1.50 & -1.28 & 1.30 & -0.70 & -2.50 & -0.77 \\
\hline 7 & 50 & 100 & 1 & 2 & 1.5 & -2.59 & -2.56 & -0.31 & -2.02 & -2.05 & -13.41 \\
\hline 8 & 50 & 100 & 1 & 2 & 2 & 0.98 & -1.18 & 1.51 & -1.73 & -1.74 & -8.12 \\
\hline 9 & 50 & 100 & 1 & 2 & 2.5 & 0.43 & -1.07 & 1.34 & -0.98 & -0.62 & -1.51 \\
\hline 10 & 50 & 150 & 0.5 & 2 & 1.5 & -1.69 & -1.17 & -1.20 & -2.04 & -2.37 & -3.72 \\
\hline 11 & 50 & 150 & 1 & 3 & 1.5 & 1.56 & 2.41 & -0.92 & -1.61 & -3.48 & -2.57 \\
\hline 12 & 50 & 200 & 1 & 4 & 1.5 & -1.85 & -0.91 & -1.14 & 1.64 & -1.92 & -1.58 \\
\hline 13 & 75 & 150 & 1 & 2 & 2 & -1.97 & -1.68 & -1.01 & -1.68 & -3.35 & -5.18 \\
\hline 14 & 75 & 225 & 1 & 3 & 2 & -1.91 & -1.39 & -1.23 & -1.11 & -3.28 & -2.97 \\
\hline 15 & 75 & 225 & 1 & 3 & 2.5 & -1.89 & -1.16 & -1.36 & -1.33 & -3.00 & -2.93 \\
\hline 16 & 75 & 300 & 1 & 4 & 3 & -2.38 & -1.28 & -2.17 & -3.29 & -2.17 & -9.86 \\
\hline
\end{tabular}

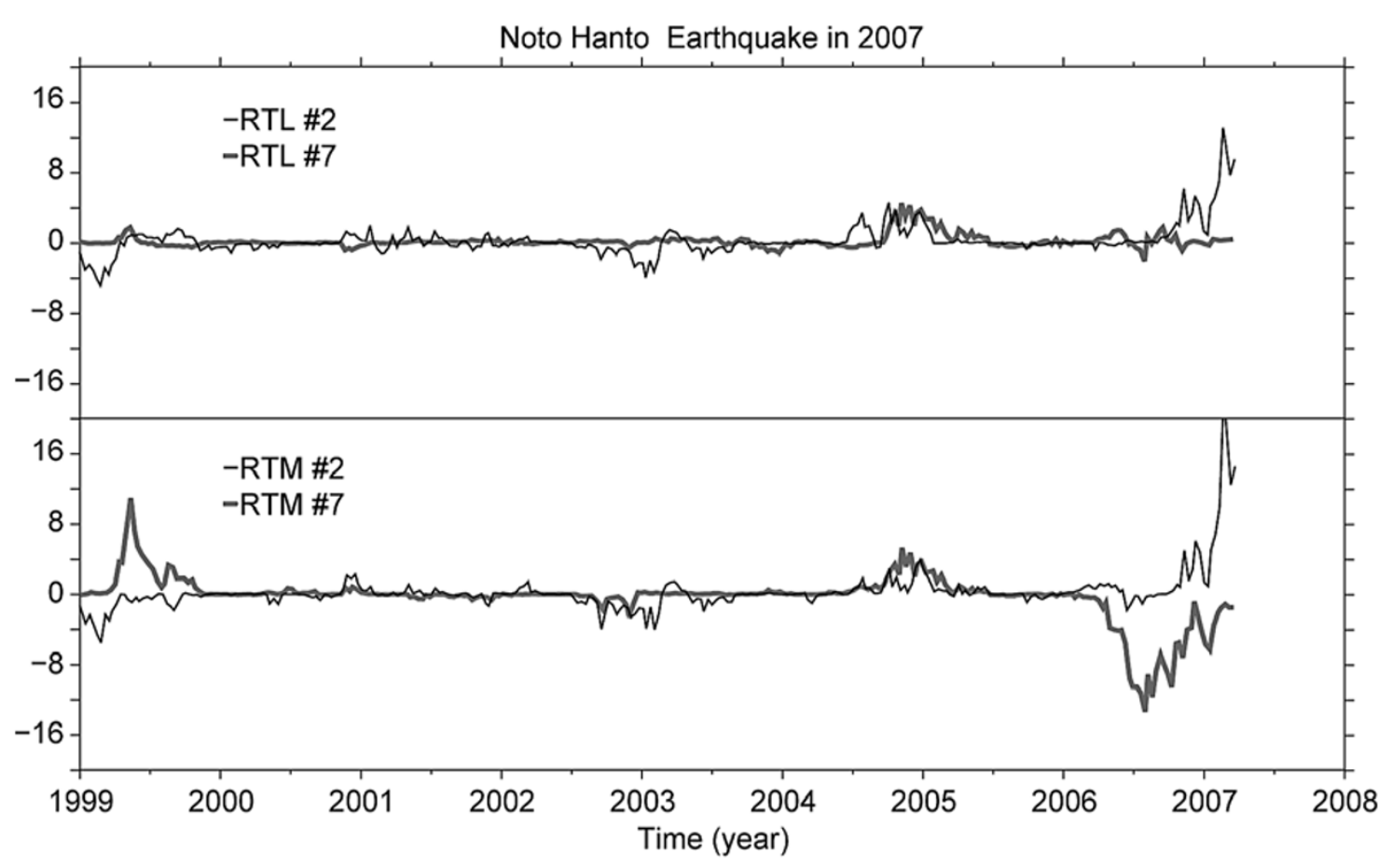

Fig. 4. Time variations of the $R T L$ and $R T M$ under the parameter sets \#2 and \#7 at the epicenter $\left(136.69^{\circ} \mathrm{E}, 37.22^{\circ} \mathrm{N}\right)$ of the Noto Hanto Earthquake in 2007 in central Japan. Upper and lower panels represent the time variation of the RTL and RTM values. Thin and thick lines indicate \#2 and \#7, respectively.

in operation. (Actually, the construction of Hi-net was motivated by this earthquake.) As demonstrated by the shaded values, the RTM algorithm seems to reveal seismic quiescence more sensitively than the RTL algorithm. Figure 3 shows the quiescence maps obtained from the RTL and RTM algorithms applied at each $0.1^{\circ}$ grid around the epicenter as of September 30, 1994. Both maps indicate that the main shock occurred on the edge of the seismic quies- 


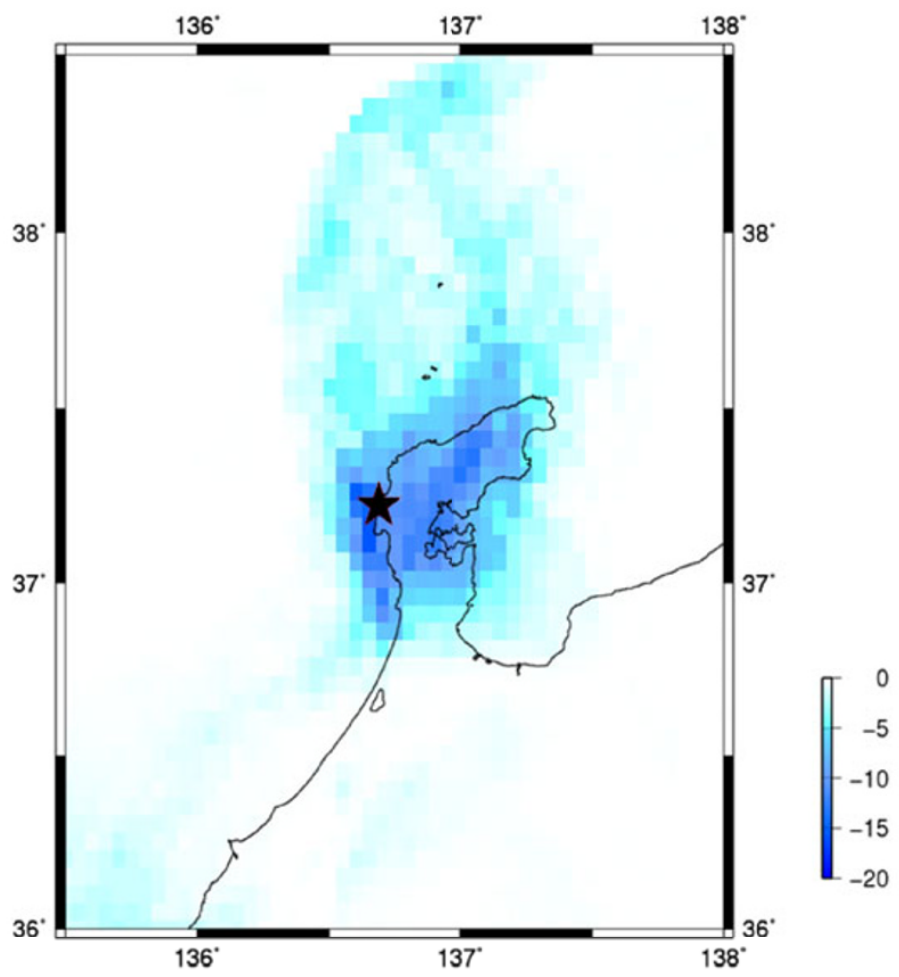

Fig. 5. The seismic quiescence map around the epicenter of the Noto Hanto Earthquake in 2007 ( $\left.M_{\mathrm{JMA}} 6.9\right)$ in central Japan when the epicenter takes the minimum $R T M$ value under the parameter set \#7 in Table 2 (July, 2006). Star shows the epicenter.

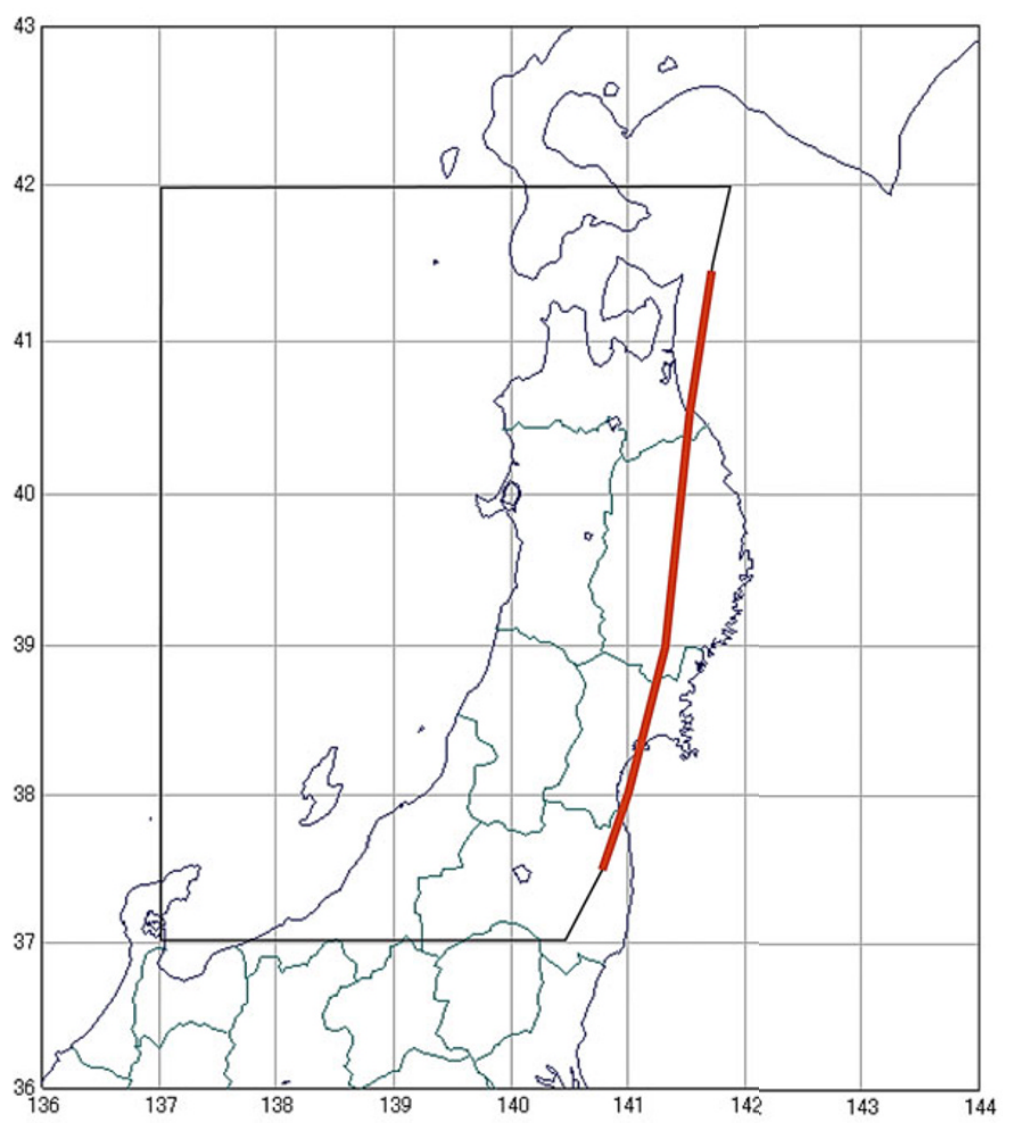

Fig. 6. The earthquakes area used for the calculation. The aseismic front (thick line) is taken into account. 
Table 3. Results of parameter survey in RTL and RTM algorithms at the epicenter of the Iwate-Miyagi Nairiku Earthquake in 2008 ( $M_{\mathrm{JMA}}$ 7.2).

\begin{tabular}{|c|c|c|c|c|c|c|c|c|c|c|c|}
\hline \multirow{2}{*}{ No. } & \multicolumn{5}{|c|}{ Parameters used for calculation } & \multicolumn{6}{|c|}{$\begin{array}{l}\text { Values at the minimum } R T L \text { or } R T M \\
\text { within } T_{\max } \text { from the occurrence time }\end{array}$} \\
\hline & $\begin{array}{l}R_{0} \\
(\mathrm{~km})\end{array}$ & $\begin{array}{l}R_{\max } \\
(\mathrm{km})\end{array}$ & $\begin{array}{l}T_{0} \\
\text { (year) }\end{array}$ & $\begin{array}{l}T_{\max } \\
\text { (year) }\end{array}$ & $M_{\min }$ & $R$ & $T$ & $L$ & $M$ & $R T L$ & $R T M$ \\
\hline 1 & 25 & 50 & 0.5 & 1 & 1.5 & -2.56 & -2.39 & -0.98 & -2.14 & -6.00 & -13.12 \\
\hline 2 & 25 & 75 & 0.5 & 1 & 1.5 & -2.70 & -2.82 & -1.00 & -2.49 & -7.61 & -18.98 \\
\hline 3 & 25 & 100 & 0.5 & 1.5 & 1.5 & -2.06 & -2.51 & -0.91 & -1.61 & -4.71 & -8.30 \\
\hline 4 & 25 & 100 & 0.5 & 2 & 1.5 & -2.30 & -1.36 & -1.10 & -1.48 & -3.47 & -5.78 \\
\hline 5 & 25 & 100 & 0.5 & 2 & 2 & -2.64 & -2.24 & -1.01 & -1.46 & -5.97 & -8.63 \\
\hline 6 & 25 & 100 & 0.5 & 2 & 2.5 & -1.78 & -0.84 & -0.91 & -0.97 & -1.36 & -1.45 \\
\hline 7 & 50 & 100 & 1 & 2 & 1.5 & -2.04 & -1.56 & -1.10 & -1.67 & -3.52 & -5.31 \\
\hline 8 & 50 & 100 & 1 & 2 & 2 & -2.22 & -2.09 & -1.01 & -1.59 & -4.70 & -7.02 \\
\hline 9 & 50 & 100 & 1 & 2 & 2.5 & -1.22 & -0.84 & -0.91 & -0.97 & -0.93 & -0.99 \\
\hline 10 & 50 & 150 & 0.5 & 2 & 1.5 & -1.65 & -1.85 & -0.84 & -1.03 & -2.55 & -3.14 \\
\hline 11 & 50 & 150 & 1 & 3 & 1.5 & -1.72 & -1.66 & -0.74 & -1.17 & -2.12 & -3.17 \\
\hline 12 & 50 & 200 & 1 & 4 & 1.5 & -2.02 & -2.15 & -0.86 & -2.05 & -3.74 & -8.89 \\
\hline 13 & 75 & 150 & 1 & 2 & 2 & -1.81 & -1.57 & -1.00 & -0.92 & -2.84 & -2.62 \\
\hline 14 & 75 & 225 & 1 & 3 & 2 & -1.12 & -1.32 & -0.68 & -1.13 & -1.09 & -1.76 \\
\hline 15 & 75 & 225 & 1 & 3 & 2.5 & -2.23 & -2.39 & -0.75 & -2.28 & -3.99 & -12.18 \\
\hline 16 & 75 & 300 & 1 & 4 & 3 & -2.78 & -1.26 & -0.67 & -1.58 & -2.36 & -3.72 \\
\hline
\end{tabular}

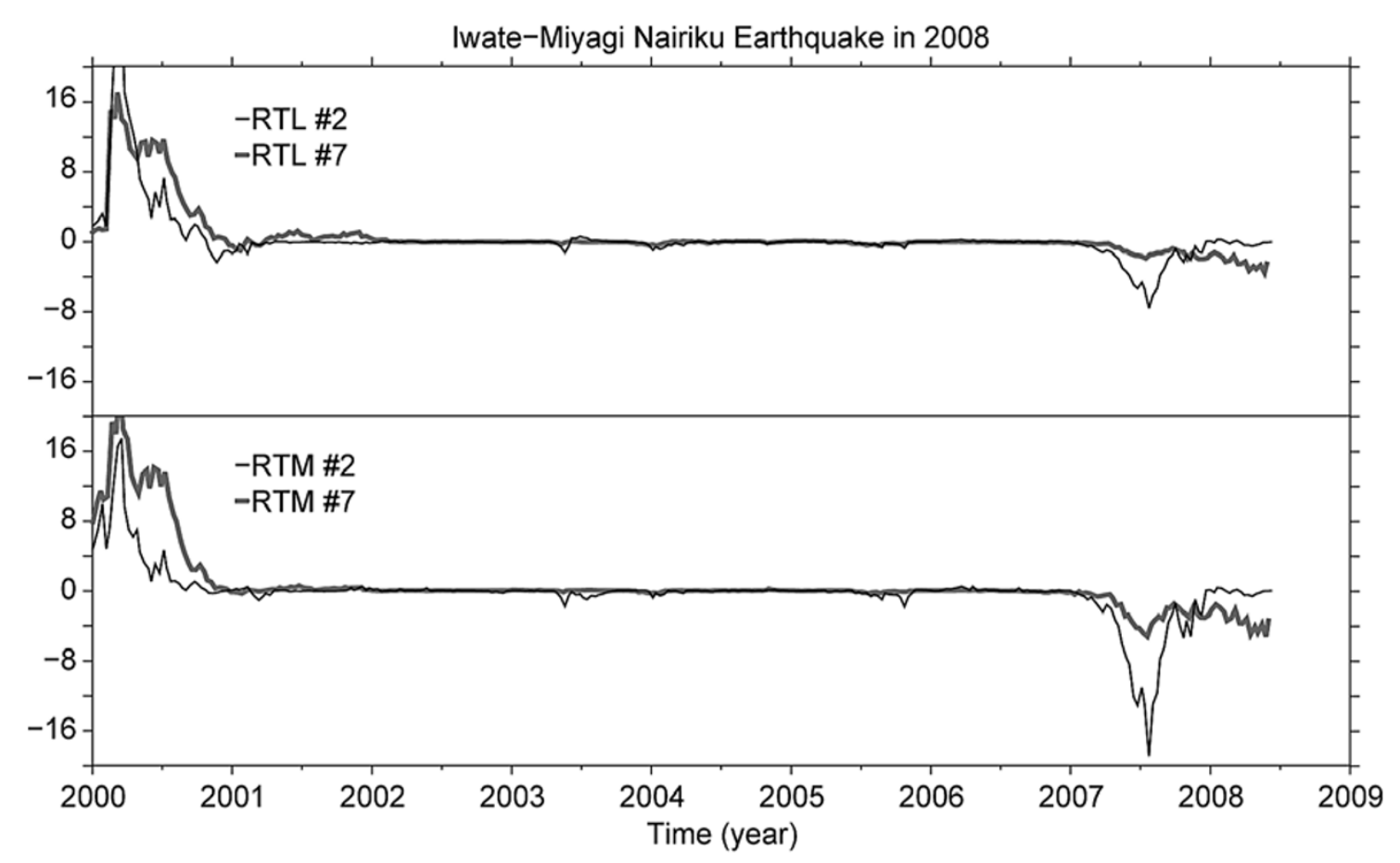

Fig. 7. Time variation of $R T L$ and $R T M$ under the parameter sets \#2 and \#7 at the epicenter $\left(140.88^{\circ} \mathrm{E}, 39.03^{\circ} \mathrm{N}\right)$ of the Iwate-Miyagi Nairiku Earthquake in 2008 (M 7.2) in northeast Japan. Upper and lower panels represent the time variation of the RTL and $R T M$ values. Thin and thick lines indicate \#2 and $\# 7$, respectively.

cence area. Huang et al. (2001) obtained a similar RTL map from the earthquake catalog with $M_{\mathrm{c}}=3.0$.

The Noto Hanto Earthquake in 2007 ( $\left.M_{\mathrm{JMA}} 6.9\right)$ occurred off the coast of Noto peninsula in central Japan on March
25,2007 . We also decluster the earthquake data from the JMA with the " $3 \mathrm{~km} / 7$ days" routine. Table 2 shows the results of the RTL and RTM parameter survey on this earthquake. The RTM algorithm indicates seismic quiescence 


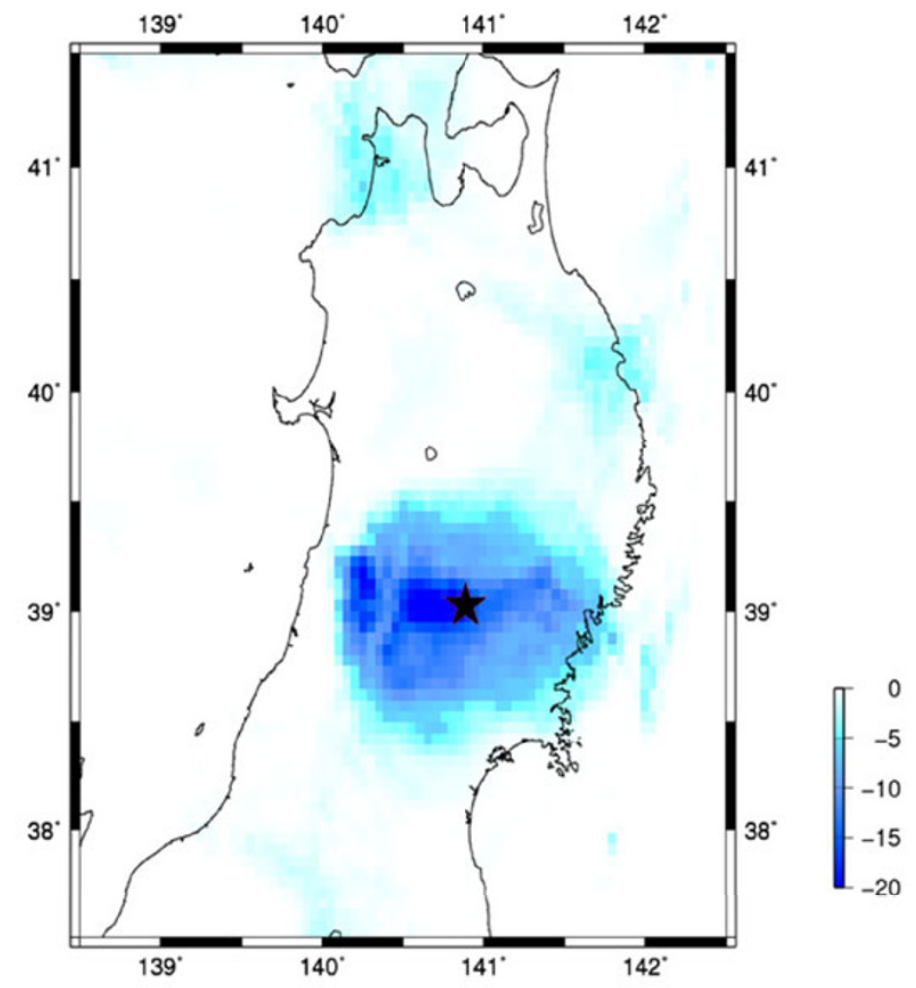

Fig. 8. The seismic quiescence map around the epicenter of the Iwate-Miyagi Nairiku Earthquake in 2008 ( $\left.M_{\text {JMA }} 7.2\right)$ in northeast Japan when the epicenter takes the minimum RTM value under the parameter set \#7 in Table 3 (July, 2007). The star shows the epicenter.

in four parameter sets (darker shading) and seismic quasiquiescence in two parameter sets (lighter shading); in contrast, the RTL algorithm indicates no quiescence. Figure 4 shows examples of the time variation of the RTL and RTM in parameter sets \#7 and \#2 of Table 2 at the epicenter $\left(136.69^{\circ} \mathrm{E}, 37.22^{\circ} \mathrm{N}\right)$. The calculation was made in the period from January 1, 1999 to 1 day before the earthquake occurrence.

Parameter set \#2 is representative of ones with the smaller $R_{\max }$ and $T_{\max }$ ranges, while parameter set \#7 is the most widely used one in early studies with the RTL algorithm (e.g., Huang et al., 2001; Huang and Nagao, 2002). In Fig. 4, the RTM algorithm together with parameter set \#7 detects the seismic quiescence before the main shock (the best set), while the RTL algorithm does not show such an anomaly. In addition, both the RTL and RTM algorithms with parameter set \#2 detect seismic activation before the main shock. This result means that local activation is often reported when there is actually local activation and quiescence simultaneously (e.g., Wyss and Habermann, 1988; Chen et al., 2005; Matsumura, 2005). Figure 5 shows the quiescence map around the epicenter as of July, 2006, about 8 months prior to the earthquake. This map is obtained from the RTM algorithm applied at each $0.1^{\circ}$ grid in parameter set \#7 in Table 2.

The Iwate-Miyagi Nairiku earthquake in 2008 $\left(M_{\text {JMA }} 7.2\right)$ occurred in northeast Japan on June 14 , 2008. There is a clear seismic activity boundary, called an aseismic front (Yoshii, 1979), lying east of the epicenter, as shown by the thick line in Fig. 6 . The seismic activity east of this boundary is due directly to the subduction of the Pacific plate and is much higher than that of the west region (e.g., Tsuji et al., 2008). The crustal seismicity on the inland side area, in which we are interested, is quite different from that of the subduction-related area. Therefore, we selected earthquakes only in the region west of the aseismic front, as shown in Fig. 6. The reason for this selection is as follows: when the $r_{i}$ range (and $R_{\max }$ ) reaches the high seismic activity region in the east from the lower seismicity region of our interest, a relatively small change of activity in the eastern region leads to a large change on the statistics in the western region. Figure 7 shows examples of the time variation in the RTL and $R T M$ in parameter sets \#2 (the best set) and \#7 of Table 3 at the epicenter $\left(140.88^{\circ} \mathrm{E}, 39.03^{\circ} \mathrm{N}\right)$. The calculation was made in the period from January 1, 2000 to 1 day before the occurrence of the earthquake. Figure 8 shows the quiescence map around the epicenter as of July, 2007, about 1 year before the earthquake. For this earthquake, in parameter set \#7, both the RTL and RTM algorithms show that the seismic quiescence stage gradually progresses until the main shock. In conclusion, the RTM algorithm clearly indicates a stronger seismic quiescence before the earthquake. While the RTL algorithm indicates only seismic quasi-quiescence in two parameter sets, the RTM algorithm indicates seismic quiescence in six parameter sets and seismic quasi-quiescence in one parameter set.

\section{Comparison with Other Methods Used for Seis- micity Diagnosis}

At this stage, it may be helpful to compare the RTL/RTM algorithms with other methods by pointing out their respec- 
tive advantages and disadvantages.

To the best of the authors' knowledge, there is no published report on seismic quiescence phenomena before the Noto Hanto Earthquake in 2007. We have now demonstrated that the RTM algorithm is able successfully detect such phenomena.

Using the $\triangle \mathrm{CFS}$ (Coulomb failure stress) calculation, Kumazawa et al. (2010) reported seismic quiescence phenomena preceding the Iwate-Miyagi Nairiku earthquake in 2008 in the regions of the stress shadow area. However, as this algorithm needs data on the focal mechanism of the main shock, it is impossible to obtain the results prior to the occurrence of the main shock. In comparison, the RTL/RTM algorithms are very simple and do not need information on focal mechanism. We believe that this is an advantage of the RTL/RTM algorithms.

The $Z$-value is one of the well-known methods to evaluate the seismicity, especially seismic quiescence phenomena. This algorithm treats the deviation from the mean seismicity, therefore the values change smoothly. In contrast, the RTL/RTM algorithms treat the product of three factors that are normalized by the background trend, which means that the RTL/RTM values are zero at the usual time. Therefore, the changes in these values tend to be clearly visible when something unusual happens.

The M8 algorithm treats multiparameters (e.g., the absolute seismicity, its deviation, and the spatial concentration of hypocenters, etc.) and routinely cautions "Times of Increased Probability of Strong Earthquakes (TIP)" when some of the parameters exceed their threshold levels. This algorithm focuses not only seismic quiescence phenomena. In this algorithm, if an expected earthquake occurs within 5 years, this caution is judged to be true. However, the period ( 5 year) is longer than that in the RTL/RTM algorithms $\left(T_{\max }\right)$.

\section{Discussion and Conclusions}

For the 1995 Kobe earthquake, the seismic quiescence does not appear when $M_{\min } \leq M_{\mathrm{c}}=2.0$ (Table 1). This absence means that the completeness in the earthquake catalog is really important for this kind of analysis.

The best parameter set is different among the three earthquakes. In the case of the 1995 Kobe earthquake (Table 1), parameter sets with the longer $T_{\max }$ and the larger $M_{\min }$ seem to be better. On the other hand, in the case of the Iwate-Miyagi Nairiku earthquake (Table 3), those parameter sets with the shorter $T_{\max }$ and the smaller $M_{\min }$ seem to be better. This difference is based on a number of complicated factors, such as the seismic event frequency, the degree of declustering, among others.

It is widely known that not all quiescence is followed by the activation of seismicity. We should evaluate the results obtained through multiparameter sets rather than rely on one parameter set as this would help avoid any acceptance of false seismic quiescence. For the three earthquakes in this study, one distinct quiescence and one major earthquake appeared during the calculation period. Further study may clarify whether or not the RTM algorithm can contribute to the development of this aspect. One possible approach to use when the aim is to clarify the statistical sig- nificance of the results would be apply calculations using many synthetic catalogs (e.g., Sobolev et al., 2002). However, we have not made this kind of statistical check since the main aim of this paper is to introduce the RTM algorithm.

Based on the results of the test applications described above, we consider that the RTM algorithm is preferable to the RTL logarithm for identifying seismic quiescence. Although it is essentially important to check the completeness of the seismic catalog (e.g., Nanjo et al., 2010a, b) as mentioned above, the present earthquake catalog in Japan is sufficiently complete to be used for seismicity analysis due to the Hi-net, the new dense seismic station network. However, for a full justification of the present conclusion, further detailed investigations on such issues as the reasonability of the proposed parameter sets and the declustering process are needed.

At this moment, whole surveyed parameters are empirically selected. Therefore, we have to consider the physical meaning of the "best fit" parameter, e.g., the relation to $\triangle$ CFS (e.g., Scholz, 1990), among others, in the future. For instance, the RTL and RTM algorithms use an exponential decay in the factors $R$ and $T$. Theoretically, seismicity is governed by the re-distribution of tectonic stress. Therefore, the factor $R$ may be as a function of $1 / r^{3}$. This area definitely needs further investigation.

In retrospective studies, we "know" the hypocenter and magnitude of "impending" earthquakes. Consequently, we can perform multiparameter surveys around the known location and magnitude. What can we do in a preseismic stage when none of this information is known? We would like to propose a study in which RTM calculations are routinely made at every grid-point of a region in order to draw a seismic quiescence map, such as those in Figs. 5 and 8, in a multiparameter survey. Computer capability will allow such a study any time now or in the near future. The experience gained in such a study may lead to a narrowing down of the ranges of parameters and, ultimately, even regional progressive diagnosis of seismic quiescence will be realized in a not so distant future. The authors consider this paper to be the first step in future analyses of seismic quiescence phenomena along the line of the RTL algorithm proposed by Gennady Sobolev in 1997.

Acknowledgments. The earthquake catalog used in this study is from JMA. We would like to thank H. Takayama of JMA for providing a declustering program. We would like to thank also A. Yoshida of JMA for his advice on decluster parameters. Our thanks are extended to S. Uyeda, Q. Huang, G. A. Sobolev, H. Katao, and K. Nanjo who gave us valuable comments and suggestions to improve the manuscript. This research was partially supported by Observation and Research Program for Prediction of Earthquakes and Volcanic Eruptions, the Ministry of Education, Culture, Sports, Science and Technology (MEXT) of Japan.

\section{References}

Chen, C.-C., J. B. Rundle, J. R. Holliday, K. Z. Nanjo, D. L. Turcotte, S.-C. Li, and K. F. Tiampo, The 1999 Chi-Chi, Taiwan, earthquake as a typical example of seismic activation and quiescence, Geophys. Res. Lett., 32(22), L22315, doi:10.1029/2005GL023991, 2005.

Frohlich, C. and S. D. Davis, Single-link cluster analysis as a method to evaluate spatial and temporal properties of earthquake catalogues, Geophys. J. Int., 100, 19-32, 1990. 
Huang, Q., Search for reliable precursors: A case study of the seismic quiescence of the 2000 western Tottori prefecture earthquake, J. Geophys. Res., 111, B04301, doi: 10.1029/2005JB003982, 2006.

Huang, Q., Seismicity changes prior to the Ms8.0 Wenchuan earthquake in Sichan, China, Geophys. Res. Lett., 35, L23308, doi:10.1029/ 2008GL036270, 2008.

Huang, Q. and T. Nagao, Seismic quiescence before the 2000 M=7.3 Tottori earthquake, Geophys. Res. Lett., 29(12), doi:10.1029/ 2001GL013835, 2002.

Huang, Q., G. A. Sobolev, and T. Nagao, Characteristics of the seismic quiescence and activation patterns before the $\mathrm{M}=7.2$ Kobe earthquake, January 17, 1995, Tectonophysics, 337, 99-116, 2001.

Kasahara, K., Earthquake Mechanics, Cambridge Univ. Press., Cambridge, 248 pp, 1981.

Keilis-Borok, V. I. and V. G. Kossobokov, Premonitory activation of earthquake flow: Algorithm M8, Phys. Earth Planet. Inter., 61, 73-83, doi:10.1016/0031-9201(90)90096-G, 1990.

Kisslinger, C., An experiment in earthquake prediction and the 7 May 1986 Andreanof Islands earthquake, Bull. Seismol. Soc. Am., 78, 218-229, 1988.

Kumazawa, T., Y. Ogawa, and S. Toda, Precursory seismic anomalies and transient crustal deformation prior to the $2008 \mathrm{Mw}=6.9$ IwateMiyagi Nairiku, Japan, earthquake, J. Geophys. Res., 115, B10312, doi:10.1029/2010JB007567, 2010.

Matsumura, S., Why does the precursory change of seismicity rate tend to be quiescence?, Zisin, 57, 441-444, 2005 (in Japanese with English Abstract).

Mogi, K., Two kinds of seismic gap, Pure Appl. Geophys, 117, 1172-1186, 1979.

Nanjo, K. Z., D. Schorlemmer, J. Woessner, S. Wiemer, and D. Giardini, Earthquake detection capability of the Swiss Seismic Network, Geophys. J. Int., 181(3), 1713-1724, doi:10.1111/j.1365246X.2010.04593.x, 2010a.

Nanjo, K. Z., T. Ishibe, H. Tsuruoka, D. Schorlemmer, Y. Ishigaki, and N. Hirata, Analysis of the completeness magnitude and seismic network coverage of Japan, Bull. Seismol. Soc. Am., 100(6), 3261-3268, doi:10.1785/0120100077, 2010b.

Obara, K., Hi-net: High sensitivity seismograph network, Japan, Lect. Notes Earth Sci., 93, 79-88, doi:10.1007/BFb0117693, 2003.

Ogata, Y., Monitoring of anomaly in the aftershock sequence of the 2005 earthquake of M7.0 off coast of the western Fukuoka, Japan, by the ETAS model, Geophys. Res. Lett., 33(1), L01303, doi:10. 1029/2005GL024405, 2006.
Rundle, J. B., K. F. Tiampo, W. Klein, and J. S. Sa Martins. Selforganization in leaky threshold systems: The influence of near-mean field dynamics and its implications for earthquakes, neurobiology, and forecasting, Proc. Natl. Acad. Sci. USA., 99, suppl. 1, 2514-2521, 2002.

Scholz, C. H., The Mechanisms of Earthquakes and Faulting, Cambridge University Press, 439 pp., 1990.

Shebalin, P., V. Keilis-Borok, I. Zaliapin, S. Uyeda, T. Nagao, and N. Tsybin, Advance short-term prediction of the large Tokachi-oki earthquake, September 25, 2003, M=8.1 A case history, Earth Planets Space, 56, 715-724, 2004.

Sobolev, G. A. and Y. S. Tyupkin, Low-seismicity precursors of large earthquakes in Kamchatka, Volcanol. Seismol., 18, 433-446, 1997.

Sobolev, G. A. and Y. S. Tyupkin, Precursory phases, seismicity precursors, and earthquake prediction in Kamchatka, Volcanol. Seismol., 20, 615-627, 1999.

Sobolev, G., Q. Huang, and T. Nagao, Phases of earthquake's preparation and by chance test of seismic quiescence anomaly, J. Geodyn., 33, 425432, 2002.

Tsuji, Y., J. Nakajima, and A. Hasegawa, Tomographic evidence for hydrated oceanic crust of the Pacific slab beneath northeastern Japan: Implications for water transportation in subduction zones, Geophys. Res Lett., 35, L14308, doi:10.1029/2008GL034461, 2008.

Wiemer, S. and M. Wyss, Seismic quiescence before the Landers $(M=7.5)$ and Big Bear (M=6.5) 1992 earthquakes, Bull. Seismol. Soc. Am., 84, 900-916, 1994.

Woessner, J. and S. Wiemer, Assessing the quality of earthquake catalogues: Estimating the magnitude of completeness and its uncertainty, Bull. Seismol. Soc. Am., 95(2), 684-698, doi:10.1785/0120040007, 2005.

Wyss, M. and R. E. Habermann, Precursory Seismic quiescence, Pure Appl. Geophys., 126, 319-332, 1988.

Wyss, M., F. W. Klein, and A. C. Johnston, Precursor ot the Kalapana M=7.2 earthquake, J. Geophys. Res., 86, 3881-3900, 1981.

Wyss, M., G. Sobolev, and J. D. Clippard, Seismic quiescence precursors to two M7 earthquakes on Sakhalin Island, measured by two methods, Earth Planets Space, 56, 725-740, 2004.

Yoshii, T., A detailed cross-section of the deep seismic zone beneath northeastern Honshu, Japan, Tectonophysics, 55, 349-360, 1979.

T. Nagao (e-mail: nagao@scc.u-tokai.ac.jp), A. Takeuchi, and K. Nakamura 